\title{
Avaliação da toxicidade ocular por derivados da 4-aminoquinolona
}

\author{
Evaluation of ocular toxicity due to 4-aminoquinoline derivatives
}

\author{
Raquel Goldhardt ${ }^{1}$ \\ Zélia Maria da Silva Corrêa ${ }^{2}$ \\ Maria Cláudia Eichenberg ${ }^{3}$ \\ Ítalo Mundialino Marcon ${ }^{4}$ \\ Antônio Vaccaro Filho
}

\section{RESUMO}

Objetivo: Avaliar a associação entre altas dosagens de derivados da 4aminoquinolona com alterações na acuidade visual, fundo de olho e campo visual. Métodos: Estudo prospectivo de 86 pacientes em uso de cloroquina ou hidroxicloroquina (Plaquinol ${ }^{\circledR}$ ) cuja visita inicial consistiu do exame da acuidade visual, biomicroscopia do segmento anterior do olho, oftalmoscopia binocular indireta, campimetria computadorizada (estímulo vermelho, programa 10.2-central), cálculo da dose diária e dose cumulativa usada pelo paciente. Consideradas doses cumulativas tóxicas de cloroquina $\geq 100 \mathrm{~g}$ e hidroxicloroquina $\left(\right.$ Plaquinol $\left.^{\circledR}\right) \geq 300$ g. Resultados: Todos os 86 pacientes examinados eram mulheres, média de idade $=45,08$ anos $(\mathrm{DP}=14,63)$, usando cloroquina $(n=13)$ ou hidroxicloroquina $(n=73)$. Verificou-se uma associação entre a exposição às drogas e a baixa da visão com significância estatística $(\mathrm{p}=0,05)$ e risco relativo $=0,3(\mathrm{IC}=0,1-1,1)$. À fundoscopia, nenhum paciente usuário de cloroquina (dose não tóxica) apresentou alteração macular e 41,7\% $(n=5)$ daqueles com doses tóxicas apresentaram alterações. No grupo da hidroxicloroquina, 21,8\% $(n=13)$ apresentaram alterações fundoscópicas com doses não tóxicas, e 17,6\% $(n=3)$ com doses tóxicas. Comparando pacientes com alterações campimétricas e aqueles sem alterações nos grupos usando cloroquina $(p=0,423)$ ehidroxicloroquina $(\mathrm{p}=0,999)$, não houve diferença estatisticamente significativa entre alteração de campo visual e dose tóxica da droga. Conclusão: A perda visual maior ocorreu nos usuários de cloroquina. Esse dado ressalta a importância da medida da acuidade visual na avaliação e acompanhamento de pacientes em uso dos derivados da 4-aminoquinolona. Não foi observada diferença significativa entre os usuários de altas e baixas doses dos derivados da 4-aminoquinolona que apresentaram alteração de campo visual.

Descritores: Retina/efeitos de drogas; Acuidade visual/efeitos de drogas; Campos visuais/efeitos de drogas; Fundo de olho; Mácula lútea/efeitos de drogas; Doenças retinianas/induzido quimicamente; Escotoma/etiologia; Hidroxicloroquina/administração \& dosagem; Hidroxicloroquina/toxicidade; Cloroquina/administração \& dosagem; Cloroquina/toxicidade; Toxicidade de drogas; Estudo comparativo

\section{INTRODUÇ̃̃̃O}

A cloroquina, derivado da 4-aminoquinolona, ficou conhecida pelo seu uso na profilaxia e tratamento da malária, e durante a 2a Guerra Mundial ${ }^{(1-2)}$. A partir de 1950, a cloroquina foi usada em doses maiores no tratamento da artrite reumatóide, lupus eritematoso sistêmico e amebíase ${ }^{(1,3)}$.

Em 1955, a hidroxicloroquina, também derivada da 4-aminoquinolona e similar à cloroquina, foi introduzida no mercado para o tratamento de doenças do colágeno (especialmente lupus eritematoso sistêmico - LES), artrite reumatóide, e doenças crônicas da pele, incluindo lupus discóide e sarcoi- 
$\operatorname{dos}^{(2)}$. Os efeitos adversos da hidroxicloroquina são semelhantes aqueles da cloroquina, mas há evidências que a incidência dos efeitos retinotóxicos são menores após o uso de hidroxicloroquina ${ }^{(4-5)}$.

A toxicidade ocular associada com agentes antimaláricos foi observada pela primeira vez em 1957 e em 1959, Hobbs e colaboradores reconheceram pela primeira vez a toxicidade retiniana associada ao uso de cloroquina ${ }^{(2-3)}$. O metabolismo dos derivados da 4-aminoquinolona é complexo e se dá pela absorção intestinal e acúmulo em tecidos como fígado, baço, pulmões e rins. Sua meia-vida aumenta proporcional à dose ${ }^{(6)}$. No olho, a cloroquina manifesta sua toxicidade graças a sua afinidade pelas estruturas pigmentadas do olho (coróide e epitélio pigmentar da retina $(\mathrm{EPR})^{(2,6)}$. Manifestações tóxicas extra-retinianas como opacidades corneanas, alterações na íris, anormalidades da motilidade, da acomodação e do cristalino são mais freqüentes com uso da cloroquina ${ }^{(2,7)}$. A toxicidade da cloroquina é devido à quebra na barreira hematorretiniana ${ }^{(8-10)} \mathrm{o}$ que não ocorre na presença do grupo hydroxi que limita a habilidade da hidroxicloroquina atravessar esta barreira. Outras mudanças desencadeadas pela cloroquina são: branqueamento dos cílios (poliose), córnea verticilata por depósitos subepiteliais, diminuição da sensibilidade corneana e paralisia dos músculos extra-oculares. O sinal mais precoce de toxicidade é a perda de campo visual paracentral que pode preceder qualquer outra anormalidade fundoscópica ou eletrofisiológica. A alteração retiniana mais precoce ocorre na área parafoveal ${ }^{(1,11)}$, pelo aumento do anel de atrófico do epitélio pigmentar da retina (EPR) contornando a fóvea. Com o comprometimento da região foveolar há perda da visão central ${ }^{(1)}$. Num estágio mais avançado, irregularidades no epitélio pigmentar da retina e retina periférica, associadas a estreitamento das veias retinianas e palidez do disco óptico assemelham-se às distrofias tapetorretinianas primárias ou retinose pigmentar ${ }^{(1,12)}$.

Há evidências de que o fator mais importante na segurança da terapêutica é a dose diária ${ }^{(12)}$. Observou-se que: 1- doses $\leq 250 \mathrm{mg} /$ dia de cloroquina, 2 - dose cumulativa $\leq 100 \mathrm{~g}$ de cloroquina, 3- doses $\leq 750 \mathrm{mg} /$ dia de hidroxicloroquina, ou 4duração do tratamento inferior a ano diminui a incidência de retinopatia ${ }^{(12)}$. Altas doses de cloroquina ou hidroxicloroquina, freqüentemente de 250 a $750 \mathrm{mg}$ administradas diariamente durante 3 anos ou dose total superior a $300 \mathrm{~g}$, costumam causar séria toxicidade ocular ${ }^{(1-2,6)}$. Publicações anteriores estabeleceram que dose segura de cloroquina é $3,5 \mathrm{mg} / \mathrm{kg} / \mathrm{dia}$ e de hidroxicloroquina, $6,5 \mathrm{mg} / \mathrm{kg} / \mathrm{dia}^{(2,8)}$.

$\mathrm{O}$ estudo da progressão da doença, devido à toxicidade retiniana por cloroquina ou hidroxicloroquina, pode ser dividida de acordo com o tempo de uso e a dosagem prescrita em:

1 - Pré-maculopatia ou pré-retinopatia: caracterizada por acuidade visual normal e escotoma no campo central com estímulo vermelho, entre 4 e 9 graus de fixação. Se a droga é descontinuada o escotoma desaparece ${ }^{(2,6)}$.

2 - Maculopatia estabelecida: caracterizada por acuidade visual levemente reduzida (6/9 a 6/12), perda do reflexo foveolar, halo parafoveal discreto e palidez do EPR. Um esco- toma central sutil para um campo visual com estímulo branco pode ser demonstrado. Estas alterações não progridem caso a droga seja descontinuada ${ }^{(2,6)}$.

3 - Maculopatia em "olho de boi": associada com redução moderada da acuidade visual (6/18 a 6/24), hiperpigmentação foveolar central circundada por uma zona despigmentada e cercada por um anel hiperpigmentado. Na angiografia aparece um "defeito em janela" que corresponde às áreas de rarefação do epitélio pigmentar ${ }^{(2,6)}$.

4 - Maculopatia severa: caracterizada por marcada redução da acuidade visual (6/36 a 6/60), "pseudo-buraco" na fóvea e extensa atrofia do EPR circunjacente ${ }^{(2,6)}$.

5 - Maculopatia em estágio final: caracterizada por severa redução da acuidade visual, marcada atrofia do EPR e visualização dos vasos sangüíneos coroídeos maiores. As arteríolas da retina podem estar atenuadas e haver acúmulo de pigmento na retina periférica ${ }^{(2,6)}$.

Há evidências experimentais que a cloroquina fica concentrada no epitélio pigmentar retiniano e ali permanece por longo período mesmo após interrupção do tratamento. O aparecimento da retinopatia pode se dar até 7 anos após interrupção do tratamento ${ }^{(13)}$.

O tempo de aparecimento da maculopatia e a dose administrada, bem como métodos de diagnóstico precoce não foram precisamente definidos nas publicações científicas disponíveis.

\section{OBJETIVO}

Avaliar a associação entre altas dosagens de derivados da 4-aminoquinolina com alterações na acuidade visual, fundo de olho e campo visual.

\section{MÉTODOS}

Foram estudados prospectivamente 86 pacientes em uso de cloroquina $(n=13)$ ou hidroxicloroquina $\left(\right.$ Plaquinol $\left.^{\circledR}\right)(n=73)$ encaminhados do ambulatório de dermatologia e avaliados no Departamento de Oftalmologia de Hospital Escola. A avaliação inicial dos pacientes consistiu de: 1- exame da acuidade visual utilizando-se a tabela Snellen (adequadamente iluminada), posicionada a 6 metros do paciente, 2- biomicroscopia do segmento anterior do olho, 3- oftalmoscopia binocular indireta sob midríase medicamentosa com tropicamida $10 \%$, 4- campimetria computadorizada (Aparelho Humphrey 745, Humphrey Instruments, San Leandro, Ca) com estímulo vermelho e programa 10.2 (central), 5- registro da dose diária usada, 6- cálculo da dose cumulativa. Foram consideradas doses tóxicas de cloroquina $\geq 100 \mathrm{~g}$ de dose cumulativa e para os de hidroxicloroquina $\left(\right.$ Plaquinol $\left.^{\circledR}\right) \geq 300 \mathrm{~g}$ de dose cumulativa. Os 86 pacientes incluídos no estudo não apresentavam outros problemas oculares.

Os dados foram analisados pelos programas EPI-INFO e SSPS. 


\section{RESULTADOS}

Os 86 pacientes examinados eram mulheres, com média de idade de 45,07 anos no grupo usuário de cloroquina $(n=13)$ e de 45,08 anos no grupo usuário de hidroxicloroquina (Plaquinol $\left.^{\circledR}\right)(n=73)$. A raça branca foi prevalecente em 65 pacientes $(75,6 \%), 10$ pacientes eram negras, 9 amarelas e 11 mulatas.

A dose cumulativa da cloroquina apresentou uma mediana (p50) de $270.000 \mathrm{mg}$, sendo que 25\% (p25) dos pacientes usavam doses menores que $116.715 \mathrm{mg}$ e $75 \%$ (p75) usavam doses acima $625.500 \mathrm{mg}$. A dose cumulativa da hidroxicloroquina apresentou uma mediana (p50) de $99.600 \mathrm{mg}$, sendo que $25 \%$ (p25) dos pacientes usavam doses abaixo de $35.800 \mathrm{mg}$ e 75\% (p75) abaixo de $261.000 \mathrm{mg}$ (Tabela 1).

A acuidade visual do OD foi na maioria (63 olhos) 73,3\% entre 0,7 e 1,0 (Snellen). A acuidade visual no OE foi $67,4 \%$ (58 olhos) entre 0,7 e 1,0 (Tabela 2).

Entretanto, se compararmos as medidas das acuidades visuais entre os dois grupos, observaremos uma diferença estatisticamente significativa $(p=0,05)$, com risco relativo de 0,3 indicando uma associação entre a exposição às drogas e a alteração na acuidade visual. Salientando a importância do exame da acuidade visual nas revisões para manter a liberação do uso da droga (Tabela 3).

A oftalmoscopia dos usuários de cloroquina em dose nãotóxicas, nenhum paciente apresentou alterações e 5/11 pacien-

\begin{tabular}{|c|c|c|}
\hline & $\begin{array}{c}\text { Dose } \\
\text { Hidroxicloroquina }\end{array}$ & $\begin{array}{c}\text { Dose } \\
\text { Cloroquina }\end{array}$ \\
\hline Percentil 25 & 35.800 & 116.715 \\
\hline Percentil 50 & 99.600 & 270.000 \\
\hline Percentil 75 & 261.000 & 625.500 \\
\hline
\end{tabular}

\begin{tabular}{|lcccccc|}
\hline \multicolumn{2}{|c|}{ Tabela 2. Acuidade visual do olho direito e olho esquerdo } \\
AV & \multicolumn{2}{c|}{ OD } & & \multicolumn{3}{c|}{ OE } \\
\cline { 2 - 4 } \cline { 6 - 7 } & Freqüência & $\%$ & & Freqüência & $\%$ \\
1,0 a 0,7 & 63 & 73,3 & & 58 & 67,4 \\
0,66 a 0,5 & 15 & 17,4 & & 17 & 19,8 \\
0,33 a 0,25 & 4 & 4,7 & & 8 & 9,3 \\
0,16 a 0,1 & 2 & 2,3 & & 1 & 1,2 \\
Menor que 0,1 & 2 & 2,3 & & 2 & 2,3 \\
\hline
\end{tabular}

tes $(41,7 \%)$ usuários de doses tóxicas apresentaram alterações. O risco relativo foi de $2,8(0,2-37,2)$ e $p=0,792$ o que não mostra associação entre achado fundoscópico comparandose doses tóxicas com não-tóxicas. Nos usuários de hidroxicloroquina em doses não-tóxicas revelou 13/56 pacientes $(23,2 \%)$ com alterações fundoscópicas. No grupo de usuários de doses tóxicas, somente $3 / 17$ pacientes $(17,6 \%)$ apresentaram alterações retinianas. O risco relativo foi de $0,8(0,3$ a 2,4$) \mathrm{e}$ $\mathrm{o} p=0,748$ não apresentando diferença significativa nos achados fundoscópicos do grupo usuário de hidroxicloroquina comparando-se doses tóxicas com não-tóxicas. Isso mais uma vez vem mostrar a importância de ser realizado exame completo nas revisões para manter a liberação do uso da droga, incluindo exame da acuidade visual.

Estes achados independem das idades dos pacientes já que conforme demonstrado acima não houve diferença significativa nas idades entre os dois grupos (Tabela 4).

Comparando-se os pacientes com campo visual alterado com os não-alterados nos grupos de hidroxicloroquina $(\mathrm{p}=0,999)$ (Tabela 5) e cloroquina $(\mathrm{p}=0,423)$ (Tabela 6), não observamos diferença estatisticamente significativa entre alteração de campo visual e dosagem da medicação, principalmente no grupo da hidroxicloroquina. Podemos observar uma tendência no grupo usuário de cloroquina a apresentar alterações quando utilizando dosagem tóxica $(p=0,423)$ se comparado com os usuários da hidroxicloroquina $(\mathrm{p}=0,999)$.

\section{DISCUSSÃO}

Uma revisão de dados sobre rastreamento visual de pacientes em uso de hidroxicloroquina sendo acompanhados a cada seis meses durante um período de dez anos concluiu que um rastreamento oftalmológico regular não é necessário se a dose diária for menor que $6,5 \mathrm{mg} / \mathrm{kg}$ e a dose cumulativa menor que $200 \mathrm{~g}^{(11)}$.

Weiner e colaboradores analisaram achados oculares em dois pacientes (49 e 60 anos) com hidroxicloroquina. $\mathrm{O}$ fundo de olho e a angiografia fluoresceínica revelaram maculopatia em olho de boi em ambos os olhos de um paciente e despigmentação perifoveal em ambos os olhos do outro paciente. Estes dois casos demonstraram que tanto a retinopatia periférica quanto a maculopatia podem se desenvolver em pacientes em uso de hidroxicloroquina por LES e que a função retiniana se mantém estável após descontinuação da droga ${ }^{(5,11)}$.

\begin{tabular}{|c|c|c|c|}
\hline & Plaquinol ${ }^{\circledR}$ & Cloroquina & $\mathbf{R R} / \mathbf{p}$ \\
\hline AV OD $1,0-0,7$ & 55 & 8 & $\mathrm{RR}=0,5(0,1-2,1)$ \\
\hline AV OD menor que 0,7 & $18(24,7 \%)$ & $5(38,5 \%)$ & $P=0,320$ \\
\hline AV OE $1,0-0,7$ & 53 & 5 & $R R=0,2(0,1-0,9)$ \\
\hline AV OE menor que 0,7 & $20(27,4 \%)$ & $8(61,5 \%)$ & $P=0,024$ \\
\hline AV geral $1,0-0,7$ & 50 & 5 & $\mathrm{RR}=0,3(0,1-1,1)$ \\
\hline AV geral menor que 0,7 & $23(31,5 \%)$ & $8(61,5 \%)$ & $\mathrm{P}=0,05$ \\
\hline $\mathrm{RR}=$ risco relativo & & & \\
\hline
\end{tabular}




\begin{tabular}{|c|c|c|c|c|}
\hline & Fundoscopia & Doses tóxicas & Doses não-tóxicas & $\mathbf{R} \mathbf{R} / \mathbf{p}$ \\
\hline Hidroxicloroquina & $\begin{array}{l}\text { Alterado } \\
\text { Não-alterado }\end{array}$ & $\begin{array}{r}3 / 17(17,6 \%) \\
14 / 17\end{array}$ & $\begin{array}{l}13 / 56(23,2 \%) \\
43 / 56\end{array}$ & $0,8(0,3-2,4)$ \\
\hline Cloroquina & $\begin{array}{l}\text { Alterado } \\
\text { Não-alterado }\end{array}$ & $\begin{array}{l}5 / 11(41,7 \%) \\
6 / 11\end{array}$ & $\begin{array}{l}0(0 \%) \\
2 / 2\end{array}$ & $2,8(0,2-37,2) \quad p=0,792$ \\
\hline
\end{tabular}

\begin{tabular}{|c|c|c|c|c|c|}
\hline \multicolumn{6}{|c|}{ Tabela 5. Grupo usuário de hidroxicloroquina } \\
\hline & \multirow[t]{2}{*}{$\mathbf{R R} / \mathbf{p}$} & \multicolumn{2}{|c|}{ CV Alterado } & \multicolumn{2}{|c|}{ CV Não Alterado } \\
\hline & & Freqüência & $\%$ & Freqüência & $\%$ \\
\hline Doses tóxicas & $R R=1,0(0,4-2,4)$ & $5 / 17$ & 29,4 & $12 / 17$ & 70,58 \\
\hline Baixas doses & $P=0,999$ & $16 / 56$ & 28,6 & $40 / 56$ & 71,42 \\
\hline $\mathrm{RR}=$ risco relativo & & & & & \\
\hline
\end{tabular}

Nesse estudo foi avaliada a associação entre doses tóxicas e as consideradas não-tóxicas de cloroquina e hidroxicloroquina. Foram comparados os achados fundoscópicos alterados, alterações no campo visual central com estímulo vermelho e acuidade visual. Observamos uma tendência na associação, no grupo usuário de cloroquina, entre alteração de campo visual e uso de doses tóxicas. Já no grupo usuário da hidroxicloroquina, esta associação não ocorre o que vem corroborar com a maior segurança e freqüência com que a droga tem sido prescrita e menos toxicidade no uso da hidroxicloroquina $\left(\mathrm{Plaquinol}^{\circledR}\right)^{(14)}$. Os achados fundoscópicos deste estudo coincidem com a literatura, mas se comparadas às alterações entre usuários de doses tóxicas e não-tóxicas não foi observada diferença significativa podendo os achados estarem relacionados com a medicação ou com a idade dos pacientes.

Este estudo não incluiu a tela de Amsler na avaliação dos pacientes porque, apesar deste ser um teste sensível em detectar escotomas paracentrais dentro dos $10^{\circ}$ de fixação, não parece detectar alterações oftalmológicas subclínicas ${ }^{(15-16)}$.

A acuidade visual nos chama a atenção já que no grupo usuário da cloroquina ela foi significativamente pior. Comparando-se as medidas das acuidades visuais entre os dois grupos foi observada uma diferença estatisticamente significativa $(\mathrm{p}=0,05)$, com risco relativo de 0,3 indicando a associação entre a exposição às drogas e a alteração na acuidade visual. Salientamos, portanto, a importância do exame da acuidade visual no acompanhamento dos pacientes em uso deste tipo de medicação.

Não foi encontrada diferença significativa entre os pacientes usuários de altas e baixas doses de derivados da 4-amino- quinolona que apresentaram campimetria alterada. Isto vem confirmar a segurança no uso da hidroxicloroquina.

A detecção de 20 pacientes (23\%) com alterações fundoscópicas e 31 pacientes (36\%) com alteração campimétrica nos alertou sobre a necessidade de reavaliações mais freqüentes, incluindo a campimetria central com estímulo vermelho, em usuários de derivados da 4-aminoquinolona para deteç̧ão mais precoce possível da maculopatia tóxica.

\section{CONCLUSÕES}

A perda visual maior ocorreu nos usuários de cloroquina. Esse dado ressalta a importância da medida da acuidade visual na avaliação e acompanhamento de pacientes em uso dos derivados da 4-aminoquinolona. Não foi observada diferença significativa entre os usuários de altas e baixas doses dos derivados da 4-aminoquinolona que apresentaram alteração de campo visual.

\section{ABSTRACT}

Purpose: To evaluate the association between high doses of 4-aminoquinolone derivatives and loss of visual acuity, visual field defects, and fundus changes. Methods: Prospective study of 86 patients in use of chloroquine or hydroxychloroquine $\left(\mathrm{Plaquinol}^{\oplus}\right.$ ) whose initial visit consisted of visual acuity testing, anterior segment slit lamp examination, indirect ophthalmoscopy, computerized perimetry (red stimulus,

\begin{tabular}{|c|c|c|c|c|c|c|}
\hline \multicolumn{7}{|c|}{ Tabela 6. Grupo usuário de cloroquina } \\
\hline & \multirow{2}{*}{\multicolumn{2}{|c|}{$\mathbf{R R} / \mathbf{p}$}} & \multicolumn{2}{|c|}{ CV alterado } & \multicolumn{2}{|c|}{ CV Não alterado } \\
\hline & & & Freqüência & $\%$ & Freqüência & $\%$ \\
\hline Doses tóxicas & \multirow{2}{*}{$R R=1,6(0,4-6,7)$} & \multirow{2}{*}{$P=0,423$} & $9 / 11$ & 81,8 & $2 / 11$ & 18,18 \\
\hline Baixas doses & & & $1 / 2$ & 50 & $1 / 2$ & 50 \\
\hline $\mathrm{RR}=$ risco relativo & & & & & & \\
\hline
\end{tabular}


program 10.2-central), calculation of the daily and cumulative dose used. Cumulative toxic doses were considered $\geq 100 \mathrm{~g}$ for chloroquine and $\geq 300 \mathrm{~g}$ for hydroxychloroquine. Results: All examined eighty-six patients were women, the average age was 45.08 years in use of chloroquine $(n=13)$ or hydroxychloroquine $(n=73)\left(\right.$ Plaquinol $\left.^{\circledR}\right)$. There was a marked correlation between the use of medication and decrease in vision $(\mathrm{p}=0.05)$ with a relative risk of 0.3 (CI 95\% 0.1-1.1). Slit lamp examination of the anterior segment was unremarkable in all patients. Fundus examination of chloroquine patients taking nontoxic doses failed to reveal any abnormalities and $41.7 \%(n=5)$ of the patients using toxic doses had fundus abnormalities. In the hydroxychloroquine group, $21.8 \%(n=13)$ had fundus abnormalities while using nontoxic doses and only $17.6 \%(n=3)$ using toxic doses presented fundus abnormalities. Comparing the patients with and without visual field defect in the chloroquine group $(p=0.423)$ and hydroxychloroquine group $(p=0.999)$, there was no statistical difference between the visual field defect and the drug dosage. Conclusion: Loss of vision was greater in chloroquine patients. This information is relevant to stress the importance of visual acuity testing to follow-up patients treated with 4-aminoquinolone derivatives. The presence of visual field defects was not statistically different between patients on high or low doses of 4-aminoquinolone derivates.

Keywords: Retina/drug effects; Visual acuity/drug effects; Visual fields/drug effects; Fundus oculi; Macula lutea/drugs effects; Retinal diseases/chemically induced; Scotoma/etiology; Hydroxychloroquine/administration \& dosage; Hydroxychloroquine/toxicity; Chloroquine/administration \& dosage; Chloroquine/toxicity; Drug toxicity; Comparative study

\section{REFERÊNCIAS}

1. Gass, J. Donald M. Toxic disease affecting the pigment epithelium and retina: chloroquine $\left(\right.$ Aralen $^{\circledR}$ ) and hydroxychloroquine (Plaquenil ${ }^{\circledR}$ ) retinopathy. In: Gass JDM. Stereoscopic atlas of macular disease: diagnosis and treatment. $4^{\text {th }}$ ed. St Louis: Mosby-Year Book; 1997. p.4557-73.

2. Weinberg DV, D'Amico DJ. Retinal toxicity of systemic drugs. In: Albert DM, Jakobiec FA, editors. Principles and practice of ophthalmology: clinical practice. Philadelphia: W.B. Saunders; 1994. p.3284-5.

3. Hobbs HE, Sorsby A, Dreedman A. Retinopathy following chloroquine therapy. Lancet 1959;2:478-80.

4. Johnson MW, Vine AK. Hydroxychloroquine therapy in massive total doses without retinal toxicity. Am J Ophthalmol 1987;104:139-44.

5. Weiner A, Sandberg MA, Gaudio AR, Kini MM, Berson El. Hydroxychloroquine retinopathy. Am J Ophthalmol 1991;112:528-34.

6. Kanski JJ. Acquired macular disorders. In: Kanski JJ editor. Clinical ophthalmology. $4^{\text {th }}$ ed. Oxford: Butterworth Heinemann; 2000. p.395-462.

7. Easterbrook M. Is corneal deposition of antimalarial any indication of retinal toxicity? Can J Ophthalmol 1990;25:249-51.

8. Blomquist PH. Screening for hydroxychloroquine toxicity. Comp Ophthalmol Update 2000;1:245-50.

9. Raines MF, Bhargava SK, Rosen ES. The blood-retinal barrier in chloroquine retinopathy. Invest Ophthalmol Vis Sci 1989;30:1726-31.

10. Rynes RI. Ophthalmologic consideration in using antimalarials in the United States. Lupus 1996;5(Suppl 1):S73-4.

11. Maturi RK, Folk JC, Nichols B, Oetting TT, Kardon RH. Hydroxychloroquine retinopathy. Arch Ophthalmol 1999;117:1262-3.

12. Weinberg DV. Toxic retinopathies. In: Yanoff M, Duker JS editors. Ophthalmology. London, UK: Mosby Internacional; 1999. p.1-4.

13.Ehrenfeld M, Nesher R, Merin S. Delayed-onset chloroquine retinopathy. Br J Ophthalmol 1986;70:281-3.

14. Fuchs FD, Wannmacher L. Outros agentes antiinflamatórios. In: Funchs D, Wannmacher L. Farmacologia clínica: fundamentos da terapêutica racional. $2^{\mathrm{a}}$ ed. Rio de Janeiro: Guanabara Koogan; 1998. p.203-6.

15. Cardoso RD, Chaves ACL, Farah ME. Maculopatias tóxico-medicamentosas e por irradiação. In: Abujamra S. Retina e vítreo: clínica e cirurgia. São Paulo: Roca; 2000. p.473-81.

16. Jones SK. Ocular toxicity and hydroxychloroquine: guidelines for screening. Br J Dermatol 1999;140:3-7.

\title{
ABO ELETRÔNICO
}

\author{
Novo site
}

Acesso: http://www.abonet.com.br 